\title{
Research on the application of VRP theory in logistics transportation
}

Ruiqi Zhu and Yunkai Zhai

School of Management Engineering, zhengzhou University

\begin{abstract}
Logistics transportation problem is a hot issue in today's research, with the diversification of transport vehicles, the complexity of the transportation network, the choice of logistics transportation route is a NP problem in combinatorial optimization. The paper discusses the relevant knowledge of VRP and physical distribution routing, such as the relationship between them, how to describe the distribution problem with VRP, how to build up mathematical model and so on and meanwhile, points out the direction of research.
\end{abstract}

\section{Introduction}

Nowadays, with the integration of the logistics industry, information technology and the trend of globalization, the whole logistics distribution occupies more and more important status in the system.Transportation system is one of the most important subsystems in the distribution system, which accounts for about $50 \%$ of the total logistics cost, so it is the first to reduce the cost of logistics distribution.Among them, whether the transportation route directly affects the distribution speed, cost and benefit, especially the determination of distribution line is a complicated system engineering. Select appropriate vehicle routing, can speed up the response to customer demand, improve service quality, enhance customer satisfaction to the logistics link, reduce service provider operating costs.Optimizing the transportation logistics and reducing the transportation cost is one of the effective ways for enterprises to improve the competitiveness of enterprises, especially in the logistics distribution enterprises. Therefore, since Danting and Ramser put forward the Vehicle Routing Problem in 1959, VRP has become a research hotspot in logistics field in recent years .

\section{Logistics distribution and VRP}

The study of VRP generally has the following assumptions:

- the material distributed can be mixed;

- the location of the individual users and requirements are known;

- From the distribution center to the transportation distance between individual users is known;

- distribution center has enough resources for distribution, and has enough transport capacity.

VRP program clearly stipulates that the number of vehicles should be dispatched to meet the constraints, models and the specific driving route of each vehicle. The implementation of VRP transport scheme can ensure on time and quality of the day to complete the transportation task, and can make the total travel at least. Visible in the logistics and transportation issues, the introduction of VRP is very necessary. 


\section{Mathematical model of VRP in logistics distribution}

\section{1 logistics distribution in the VRP description}

A distribution center offers logistics services to a certain geographical scope (customer demand),amount of goods required each of demand points are small (less than the capacity of the vehicle), and each demand point distribution center as well as the needs of the distance between points is known. If the amount of goods distribution center can meet the needs of the customers, and the distribution center can cooperate with transportation companies through its own or rent or the way enough capacity available for deployment, a load weight per delivery trucks exceeds its rated load, and the total running distance of each vehicle has a certain limit.

In order to improve the utilization rate of vehicles, how to arrange the vehicle routing and vehicle scheduling can meet the distribution task, and make the total mileage of the vehicle to be the shortest. That is to say, in order to complete the transport task, the distribution center should send a plurality of cars, all delivery routes for several major routes (loop); each delivery trucks starting from the distribution center, along a route covering several users (loop) delivery, and then return to the distribution center. At this time, including our problem to be solved: what customers should be assigned to a loop (that is, what the customer goods should be arranged in the same car); bypass order on each route of customers.

\subsection{Mathematical model of VRP in logistics distribution}

A typical VRP model can be used to express the loop, you can also put the time, distance, cost into transportation costs, the basic principles are the same. Here we use the loop representation.

basic conditions. Existing n vehicles parked in a common source point (that is, the logistics center) O, it needs to provide goods to m customers. Assuming the customer is a1, a2,.., am, and the source and customer locations are known.

model target. Determine the required number of vehicles N, and assign these vehicles to a loop, including the routing and scheduling within the loop, so that the total transport distance $\mathrm{S}$ minimum.

constraint conditions.

$-\mathrm{N} \leqslant \mathrm{n}$;

- Use $Q_{i j}$ to express the first $i$ car in its sub circuit corresponding to the requirements of the first $j$ customer. Due to the previous assumptions, the number of customer demand $\mathrm{Q}_{\mathrm{ij}}$ smaller, so the customer demand is not likely to exceed the weight of the car. Therefore, each customer can only accept a car at the same time service. A sub circuit corresponding to a car, let the number of customers of each loop is 1 , i is the vehicle corresponding to the number, $\mathrm{c}_{\mathrm{i}}$ said the $\mathrm{i}$ collection by the path corresponding to the car, $\mathrm{c}_{\mathrm{ij}}$ said the order of the corresponding sub circuit for $\mathrm{j}$ customers. If $\mathrm{l}_{\mathrm{i}}=0$, then said the car did not participate in the service. From this we can know :

$$
\sum_{i=1}^{n} 1_{i}=m
$$

- After the vehicle is completed, the task is to return to the source $\mathrm{a}_{0}$.

- The vehicle can not exceed the maximum load $\mathrm{W}_{\mathrm{i}}$ and maximum running length limit of $\mathrm{I}_{\mathrm{i}}$.

mathematical model. Assume that $d_{i(j-1) j}$ between the $j-1$ car the corresponding line in the 1 customer and $j$ customer distance; $d_{i(L I)(0)}$ between the $1_{i}$ client and said the source $i$ car in the distance of the route of the corresponding $v_{0}$. The mathematical model can be established as follows: 


$$
\begin{aligned}
& \min s=\sum_{i=1}^{N}\left[\sum_{j=1}^{l_{1}} d_{i(j-1) j}+d_{i\left(l_{i}\right)(0)} \cdot \operatorname{sign}\left(l_{i}\right)\right] \\
& \text { s.t. } \quad \sum_{j=1}^{l_{i}} Q_{i j} \leq w_{i} \\
& \sum_{j=1}^{l_{1}} d_{i(j-1) j}+d_{i\left(l_{i}\right)(0)} \cdot \operatorname{sign}\left(l_{i}\right) \leq L_{i} \\
& 0 \leq l_{i} \leq m \\
& \sum_{i=1}^{N} l_{i}=m \\
& c_{i}=\left\{c_{i j} \mid c_{i j} \in\left\{v_{1}, v_{2}, \ldots, v_{n}\right\}, j=1,2, \ldots, l_{i}\right\} \\
& c_{i} \cap c_{j}=\Phi, \forall i \neq j \\
& \operatorname{sign}\left(l_{i}\right)=\left\{\begin{array}{l}
1, l_{i} \geq 1 \\
0, \text { 其他 }
\end{array}\right. \\
& N \leq n
\end{aligned}
$$

(1)the objective function, that is, the total running distance of the vehicle in the completion of the distribution task is the shortest.

(2)the vehicle capacity constraints, that is, the total demand for each sub circuit customer does not exceed the maximum load of vehicles, to ensure that the needs of all customers can not exceed the vehicle's total weight of the vehicle itself.

(3) the operating length of the vehicle in each sub circuit is not more than the maximum mileage of the vehicle.

(4) the number of customers per vehicle is not more than the total number of customers.

(5)the total number of customers involved in the operation of the vehicle, the total number of customers and the actual number of customers are equal, that is, to ensure that each customer service.

(6) the corresponding customer set for each vehicle service.

(7) each customer can only be served by a car at the same time.

(8) the i car is involved in the service.

\section{Logistics distribution in the VRP algorithm}

\subsection{Basic algorithm of Hamilton map}

Traveling salesman problem with the theory of graph theory, that is, in a complete graph of a weighting, to find a minimum weight of the Hamilton circle, said the circle as the optimal circle. At present, there is no effective algorithm to solve the problem of minimum Hamilton circle, only to find a better algorithm to solve the problem. One possible way is to first ask for a Hamilton turn $\mathrm{C}$, and then modify the $\mathrm{C}$ appropriately to get another Hamilton loop with the smaller weight. After a series of changes in the series, and finally get a circle, can not be improved by this method. This last lap is almost certainly not optimal, but there are reasons to think that it is often relatively good. In order to get a higher precision, this program can be repeated several times, each time from different circles to start. It can be said that the use of Hamilton to solve the smallest circle to find a relatively simple type of TSP or VRP.

\subsection{Other classical algorithms}

Solving the vehicle routing problem is very much, basically can be divided into 2 categories: accurate algorithm and heuristic algorithm. The exact algorithms for solving VRP problems include the branch and bound method, the cut plane 
method, the dynamic programming method, the network flow algorithm, etc.. Heuristic algorithm mainly has saving method, scanning method, two stage algorithm, tabu search algorithm, genetic algorithm, duplicate matching, etc..

Branch and bound method. The basic idea of branch and bound method to solve the VRP problem, with no integer constraints to the VRP problem (B) optimal solution as the starting point, if the solution is an integer solution, then the solution is the original VRP problem (A) of the optimal solution, otherwise B non integer solutions of adjacent integer additional conditions the formation of 2 branches, namely, 2 sub problems are solved. If the above 2 sub problems are solved, the optimal solution is the integer solution, then stop the branch of the sub problem, or continue to solve the problem. This method can only be applied to solve the small VRP problem. Kolen at alhas used this method to solve the problem of vehicle traveling with time windows, and found that when the nodes are expanded to 12 , the computer has a memory problem.

Dynamic programming method. The basic idea of dynamic programming method to solve the VRP problem, the VRP problem is considered as a decision problem of phase $\mathrm{n}$, and then transformed into decision problems in solving $\mathrm{N}$ with the recursive relation of single stage, so as to simplify the calculation process. The optimal solution of VRP can be obtained by this method, but it is only suitable for small scale optimization problems.

Two stage heuristic algorithm. Christofides, Mingozzi, Toth in 1979 proposed two stage heuristic algorithm, in order to improve the construction algorithm to solve the problem. The first phase of the algorithm commonly used heuristic algorithm to obtain a feasible solution, second stage method, Insert method、2- exchange、2- swap、2-OPT、3- OPT and other technical improvements, the adjustment in the solution remain feasible, trying to close to the optimal target, each step produces another feasible the solution to replace the original solution, the objective function is improved, continue to improve the objective function so far. Two stage method in the process of solving, often using interactive optimization technology, the person's subjective initiative to join the VRP problem solving process.

Tabu search algorithm. The idea of tabu search is proposed by Glover in 1986. It is a kind of global optimization algorithm. It introduces a tabu list, local records have searched the advantages of using tabu table information in a search or not selectively search these points, in order to jump out of the local advantages, so as to realize the global optimization. In 1994, Garcia et al firstly applied the tabu search algorithm to the VRPTW problem. Tabu search algorithm is used to solve the VRP problem, although it can ensure the exploration of different effective search methods, but it is not easy to implement in practice because it involves complex neighborhood transformation and solving strategies.

Simulated annealing method. Simulated annealing is proposed by Metropolis in 1953, and it is a kind of random search algorithm based on the principle of thermodynamics. The simulated annealing algorithm for VRP problem is studied in 1993, and the simulated annealing method is proposed to solve the Osman problem. When the VRP problem is solved by simulated annealing, the energy of the atoms in the physical annealing is equal to the distribution of the optimal node, and the atomic vibration simulation is a random search in the search space of the line. The search process is completed by the execution of the pairwise exchange of the distribution, the initial solution for the distribution of the total cost of the distribution. In order to find the optimal route, the distribution of the distribution is improved by using arbitrary pair wise exchange in the existing allocation. Simulated annealing algorithm is suitable for large-scale demand fixed or random demand of the VRP problem. In theory, the global optimal solution can be obtained by this method, but in practical application, due to the limitation of the computation time, the optimal solution of the VRP problem can only be given. In order to make the approximate solution obtained by the simulated annealing algorithm more accurate, the simulated annealing method is repeated, and the optimal solution is selected as the final approximate optimal solution.

Genetic algorithm. Genetic algorithm is a kind of improved algorithm for local search. When genetic algorithm is used to solve the VRP problem, it is mainly used to search for the common character between the biological evolutionary generation and the optimal route, and to search the optimal solution of the distribution route in the global scope. The same expenses in case of the same level distribution node, are considered to be involved in the next step of iteration, and by subsequent steps gradually eliminated, and ultimately determine the optimal delivery route; different expenses in case of 
the same level distribution node, "the fittest survive", to get the right distribution by iteration branch to spend less, but can not determine the optimal qualifications, must participate in the subsequent elimination step, until the end of the iteration, the optimal distribution route. Genetic algorithm has good robustness, flexibility and versatility, and is especially suitable for solving large scale VRP problems. But because of some defects still exist in the algorithm itself, such as premature convergence, poor local search ability, therefore, can not guarantee the maximum probability of convergence to the global optimal solution. Zhang Liping et al in 2002 by introducing a novel crossover operator to construct a kind of improved genetic algorithm. This algorithm gets rid of the diversity of population, there is no common traditional genetic algorithm premature convergence problem, optimization can effectively obtain the solution of VRP. Lang Maoxiang in 2006 through the construction of partheno genetic algorithm, effectively improved the traditional genetic algorithm for complex VRP problem of low search efficiency and easy to fall into the defects of "premature convergence".

Ant colony algorithm. Ant colony algorithm is a kind of intelligent optimization algorithm based on swarm intelligence proposed by Dorigo. The basic principle of the algorithm is to simulate the behavior of ants in search of food: ants find food, they can always find a path from food to the nest between the optimal path. This is because ants in the search path, will be released on the path of a special pheromone. When they come across a road that has not yet been passed, they randomly pick a path and release the pheromone that is related to the length of the path. The longer the path, the lower the release of the hormone concentration. When the later came to the intersection of the ants, the choice of higher probability of hormone concentration path will be relatively large. This forms a positive feedback, the optimal path of the hormone concentration is more and more large, and the other path on the hormone concentration will be decreased with the passage of time, and ultimately the entire ant colony will find the optimal path. The algorithm has a good effect in solving the VRP problem, but it is easy to fall into the local optimum problem if the calculation time is long. Huang Lan et al. In 2002, the specific application of ant algorithm in the traveling salesman problem is presented, and the problem is solved and the local optimization is solved by the opt 3-method and the crossover strategy. Hao Jin and others in 2002 for the basic ant colony algorithm, the design of a novel random perturbation ant colony algorithm, and its application to solve complex TSP problems. Wu Jianjun and others in 2004 for the logistics distribution routing problem, proposed a hybrid ant colony algorithm to overcome the classical ant colony algorithm time complexity is too difficult.

Particle swarm optimization algorithm. PSO algorithm is an evolutionary computation technique based on swarm intelligence, which was proposed by Kennedy and Eberhart in 1995. The algorithm is to solve the optimization problem by simulating the foraging behavior of birds. Firstly, in the solution space, the birds are randomly initialized, each bird is called "particle", these "particles" move in the solution space, and find the optimal solution after several iterations. In each iteration, the particle updates itself by tracking the 2 "extremes". The first one is the optimal solution of the particle itself ("Pbest"); the other extreme is the optimal solution ("Obest"), which is found in the whole particle swarm. Find these 2 extreme, each particle according to their own flight speed, determine their own direction and flight distance. PSO algorithm to solve the VRP problem with fast convergence speed, dependent on the experience of small parameters, simple, easy to use and so on. But like other evolutionary optimization algorithms, it is easy to fall into the local optimum. Huang Lan et al. By introducing the concept of exchange and exchange order in 2003, a special particle swarm optimization algorithm is constructed to solve the traveling salesman problem. Xiao Jianmei designed a new real number encoding scheme in 2005 , the vehicle routing problem is transformed into a quasi continuous optimization problem, and the penalty function method to deal with constraints, so as to solve the particle swarm algorithm is applied to the vehicle routing problem.

\section{Comparison of various basic algorithms}

Exact algorithm is based on strict mathematical means of the algorithm, in the case can be solved, the solution is usually better than the artificial intelligence algorithm. However, after the introduction of strict mathematical methods, it can not avoid the exponential explosion problem, so that the class algorithm can only solve the determination of small and 
medium scale VRP. Specific to each algorithm, they have their scope of application and characteristics. Given the lower bound and the branch and bound algorithm is related to the point of access from the perspective of the establishment, therefore is suitable for symmetric VRP, also applies to non symmetric VRP; three subscript vehicle flow equations in the model introduces effectively represents the time window variables, thus the utility model is applicable to general assignment problem (GAP) and with time window TSP (TSPTW); two subscript vehicle flow equations by SYM equation and the expansion of TSP, the removal of the number of vehicles on behalf of the subscript, the form is more compact, with fewer constraints, so only applies to symmetric CVRP and DVRP, but also for the two star to ask VRP loose constraints. $\mathrm{K}$ is thus the method is applicable to CVRP and DVRP; the feasibility conditions of tabu search are also available, so the scope of application and the two stage method: repeat the same matching algorithm in the model considering the time constraints and capacity constraints, so it is suitable for strong constraint VRP.

Deterministic VRP is the basis of the research of non deterministic VRP. At present, our country is still in the initial stage of complex VRP research. But in real life the most common or uncertain VRP, most of the uncertainty of VRP is the simplification of uncertainty VRP. The existing SVRP results have been able to solve the small scale of SVRP, but can not be competent for the larger scale of SVRP. A practical way to deal with large scale SVRP is still the artificial intelligence method, but to get a general efficient algorithm is difficult, so we should study and different algorithms with different neighborhood feature problems with neighborhood features. For FVRP, the fuzzy possibility theory should be combined with the research.

\section{References}

1. Pan Lijun, Fu Zhuo, Liu Ximei. The open vehicle routing problem with working time and time windows. computer engineering. 2014 (04)

2. Zhu Yongli, Li Kai. Research and application of multi objective model of B2C e-commerce logistics distribution vehicle routing optimization problem. Chinese business world (the second half). 2015 (12)

3. Yu Lei. The problem and reason of the distribution of $\mathrm{Y}$ in the Anhui branch. express company logistics engineering and management. 2013 (05)

4. Wang Jun, Guo Tingting. Research on Logistics Distribution Routing Based on improved ant colony algorithm. value engineering. 2013 (02)

5. Liu point, Mao Chao. E-commerce distribution center location problem and optimization. system engineering. 2015 (10) 